\title{
NORMAL PARTITIONS OF IDEMPOTENTS OF REGULAR SEMIGROUPS
}

\author{
P. G. TROTTER
}

(Received 8 June 1977; revised 14 October 1977)

Communicated by T. E. Hall

\begin{abstract}
A characterization is provided here for any normal partition of the set of idempotents of a regular semigroup $S$. As a by-product of the method used, a new characterization of the greatest congruence on $S$ corresponding to a given normal partition of its idempotents is obtained.
\end{abstract}

Subject classification (Amer. Math. Soc. (MOS) 1970): 20 M 10.

\section{Introduction}

Any congruence on a regular semigroup $S$ induces a partition, called a normal partition, of its set of idempotents $E_{S}$. In her doctoral dissertation, Feigenbaum (1975) provided a characterization for any congruence on $S$ and for any normal partition of $E_{S}$. The aim here is to make use of the sandwich sets of Nambooripad (1974) to obtain a simpler characterization of a normal partition of $E_{S}$. As a by-product of the method used, an alternative characterization of the greatest congruence on $S$ corresponding to a given normal partition of $E_{S}$ is obtained.

\section{Definitions and preliminary results}

In all that follows let $S$ denote a regular semigroup and let $E_{S}$ be its set of idempotents. For $e, f \in E_{S}$ the sandwich set of $e, f$ is

$$
S(e, f)=\left\{g \in E_{S} ; g e=g=f g, e g f=e f\right\} .
$$

For $a \in S$ let $V(a)$ denote the set of inverses of $a$. The following lemma is taken from Nambooripad (1974) and Clifford (1974).

LeMma 1.1. Suppose $e, f, h, k \in E_{S}, a, b \in S, a^{\prime} \in V(a)$ and $b^{\prime} \in V(b)$. Then

(i) $S(e, f) \neq \square$;

(ii) if $e \mathscr{L} h$ and $f \mathscr{R} k$ then $S(e, f)=S(h, k)$;

(iii) if $g \in S\left(a^{\prime} a, b b^{\prime}\right)$ then $a g b=a b$ and $b^{\prime} g a^{\prime} \in V(a b)$.

In the light of (ii) and (iii) of the lemma, define $S(a, b)=S\left(a^{\prime} a, b b^{\prime}\right)$ for any $a^{\prime} \in V(a)$ and $b^{\prime} \in V(b)$. 
LEMMA 1.2. Let $\tau$ be a congruence on $S, a, b \in S, a^{\prime} \in V(a)$ and $b^{\prime} \in V(b)$.

(i) If $a \tau \in E_{S / \tau}$ then $S(a, a) \subseteq a \tau$.

(ii) If $a \mathscr{H} b$ and $b^{*}=a^{\prime} a b^{\prime} a a^{\prime}$ then $a^{\prime} \mathscr{H} b^{*}$ and $b^{*} \in V(b)$.

Proof. By Lemma 1.1(iii) $a^{\prime} S(a, a) a^{\prime} \subseteq V\left(a^{2}\right)$. If $a \tau=\left(a^{2}\right) \tau$ then

$$
S(a, a)=a a^{\prime} S(a, a) a^{\prime} a \subseteq a V\left(a^{2}\right) a \subseteq a \tau .
$$

Thus (i) is verified. (ii) follows directly from the observation that $a \mathscr{R} b$ if and only if $a a^{\prime} b b^{\prime}=b b^{\prime}, b b^{\prime} a a^{\prime}=a a^{\prime}$ and $a \mathscr{L} b$ if and only if $a^{\prime} a b^{\prime} b=a^{\prime} a, b^{\prime} b a^{\prime} a=b^{\prime} b$.

Let $\pi$ denote an equivalence relation on $E_{S}$ and let $P_{\pi}=\left\{e \pi ; e \in E_{g}\right\} . P_{n}$ is a normal partition of $E_{S}$ if and only if there is a congruence $\tau$ on $S$ so that the restriction of $\tau$ to $E_{\mathrm{S}}$ is $\pi$. Then $P_{n}=\left\{e \tau \cap E_{S} ; e \in E_{\mathrm{S}}\right\}$.

Feigenbaum (1975) proved that a partition $P_{n}$ of $E_{g}$ is a normal partition if and only if for any $e_{i} \in E_{S}, x_{i}, y_{i} \in S^{1}$ where $i=1, \ldots, n$, so that $x_{0}\left(e_{0} \pi\right) y_{0} \cap E_{S} \neq \square$, $x_{n}\left(e_{n} \pi\right) y_{n} \cap E_{S} \neq \square$ and $x_{j}\left(e_{j} \pi\right) y_{j} \cap x_{j+1}\left(e_{j+1} \pi\right) y_{j+1} \neq \square$ for $j=0, \ldots, n-1$, then there exists $e \in E_{S}$ so that $\left(x_{0}\left(e_{0} \pi\right) y_{0} \cup x_{n}\left(e_{n} \pi\right) y_{n}\right) \cap E_{S} \subseteq e \pi$.

We will consider a partition $P_{\pi}$ of $E_{S}$ to be a partial groupoid under the partial binary operation $*$ defined by $e \pi * f \pi=g \pi, e, f, g \in E_{S}$, if and only if

$$
\square \neq(e \pi)(f \pi) \cap E_{B} \subseteq g \pi .
$$

Define a partial operation by $S$ on $P$ by $e \pi^{c^{\prime}}=g \pi, e, g \in E_{S}, c \in S, c^{\prime} \in V(c)$, if and only if $\square \neq c^{\prime}(e \pi) c \cap E_{S} \subseteq g \pi$.

A partition $P_{\pi}$ of $E_{B}$ is an $N$-partition if and only if for each $e, f \in E_{B}, c \in S$ and $c^{\prime} \in V(c)$ then

(1) $e \pi * f \pi \supseteq S(e f, e f)$ or $(e \pi)(f \pi) \cap E_{S}=\square$ and

(2) $e \pi^{\prime} \supseteq S\left(c^{\prime} e c, c^{\prime} e c\right)$ or $c^{\prime}(e \pi) c \cap E_{S}=\square$.

By Lemma 1.2(i) it can be seen that a normal partition of $E_{\delta}$ is an $N$-partition. The converse will be proved in the next section.

Given a partition $P_{\pi}$ of $E_{S}$ define relations $\mathscr{R}_{\pi}$ and $\mathscr{L}_{n}$ on $S$ by

$$
\mathscr{R}_{\pi}=\left\{(a, b) \in S \times S ;\left(a a^{\prime}\right) \pi *\left(b b^{\prime}\right) \pi=\left(b b^{\prime}\right) \pi,\right.
$$

and

$\left(b b^{\prime}\right) \pi *\left(a a^{\prime}\right) \pi=\left(a a^{\prime}\right) \pi$ for some $a^{\prime} \in V(a)$ and $\left.b^{\prime} \in V(b)\right\}$

$$
\mathscr{L}_{n}=\left\{(a, b) \in S \times S ;\left(a^{\prime} a\right) \pi *\left(b^{\prime} b\right) \pi=\left(a^{\prime} a\right) \pi,\right.
$$

$\left(b^{\prime} b\right) \pi *\left(a^{\prime} a\right) \pi=\left(b^{\prime} b\right) \pi$ for some $a^{\prime} \in V(a)$ and $\left.b^{\prime} \in V(b)\right\}$.

Note that $a \mathscr{R}_{n} a a^{\prime} \mathscr{R}_{n} a a^{*}$ and $a \mathscr{L}_{n} a^{\prime} a \mathscr{L}_{n} a^{*} a$ for any $a^{\prime}, a^{*} \in V(a)$.

LeMma 1.3. Let $P_{n}$ be an $N$-partition of $E_{\beta}$ and $e, f \in E_{\delta}$. Then

(i) $e \mathscr{R}_{\pi} f\left[e \mathscr{L}_{n} f\right]$ if and only if $S(e, f) \subseteq e \pi[f \pi]$ and $S(f, e) \subseteq f \pi[e \pi]$;

(ii) $\mathscr{R}_{\pi}$ and $\mathscr{L}_{\pi}$ are transitive relations. 
Proof. Suppose $S(e, f) \subseteq e \pi$ and $S(f, e) \subseteq f \pi$. Since $f S(e, f)=S(e, f)$ and $e S(f, e)=S(f, e)$ then $f \pi * e \pi=e \pi$ and $e \pi * f \pi=f \pi$ so $e \mathscr{R}_{\pi} f$.

Conversely suppose $e \mathscr{R}_{\pi} f$ and $p \in S(e, f)$. Choose $q \in S(e f, e f), r \in S(q, p)$ and $t \in S(q p, q p)$. Note that by Lemma 1.1(iii), $p \in V(e f)$ and $r \in V(q p)$. By the definition of sandwich sets we have $p e=p=f p$ so $p(e f)=p f,(e f) p=e p$ and similarly $r(q p)=r p,(q p) r=q r$. So $S(e f, e f)=S(p f, e p)$ and $S(q p, q p)=S(r p, q r)$. Hence $q p f=q=e p q$ so $e q=q$, and then $t=q r t=e q r t=e t$. Note that $e p q e$ and $e p$ are idempotents. Since $e \mathscr{R}_{\pi} f$ we have by condition (1) that $q \pi=e \pi * f \pi=f \pi$ and then $p \pi=(f p) \pi=f \pi * p \pi=q \pi * p \pi=t \pi$. Hence $(e p) \pi=e \pi * p \pi=e \pi * t \pi=t \pi=p \pi$. So $(e p q e) \pi=(q e) \pi=q \pi * e \pi=f \pi * e \pi=e \pi$. But then

$$
(e p q e) \pi=(e p) \pi *(q e) \pi=p \pi * e \pi=(p e) \pi=p \pi .
$$

Thus $p \pi=e \pi$. Similarly $S(f, e) \subseteq f \pi$. Thus (i) is proved.

Assume $a \mathscr{R}_{\pi} b \mathscr{R}_{\pi} c$. Then $a a^{\prime} \mathscr{R}_{\pi} b b^{\prime} \mathscr{R}_{\pi} b b^{*} \mathscr{R}_{\pi} c c^{\prime}$ for some $a^{\prime} \in V(a), b^{\prime}, b^{*} \in V(b)$ and $c^{\prime} \in V(c)$. Choose $p \in S\left(b b^{*}, c c^{\prime}\right), q \in S\left(b b^{\prime}, p\right)$ and $r \in S\left(a a^{\prime}, q\right)$. By (i) then $p \pi=\left(b b^{*}\right) \pi, q \pi=\left(b b^{\prime}\right) \pi$ and $r \pi=\left(a a^{\prime}\right) \pi$. Also $c c^{\prime} p=p, p q=q$ and $q r=r$ so $c c^{\prime} r=r$. Hence $\left(c c^{\prime}\right) \pi *\left(a a^{\prime}\right) \pi=\left(a a^{\prime}\right) \pi$ and similarly $\left(a a^{\prime}\right) \pi *\left(c c^{\prime}\right) \pi=\left(c c^{\prime}\right) \pi$. Dually $\mathscr{L}_{n}$ is transitive.

For a partition $P_{\pi}$ of $E_{S}$ define a relation $\mathscr{H}_{n}$ on $S$ by $\mathscr{H}_{n}=\mathscr{R}_{n} \cap \mathscr{L}_{n}$.

Note that if $P_{\pi}$ is a normal partition of $E_{S}$ induced by a congruence $\tau$ on $S$ then $a \mathscr{R}_{\pi} b$, or $a \mathscr{L}_{\pi} b$, or $a \mathscr{H}_{n} b$ if and only if $a \tau \mathscr{R} b \tau$, or $a \tau \mathscr{L} b \tau$, or $a \tau \mathscr{H} b \tau$ respectively.

LeMma 1.4. Let $P_{\pi}$ be an $N$-partition of $E_{S},(e, f) \in \pi$ and $c \in S$. Then ec $\mathscr{H}_{n} f c$ and ce $\mathscr{H}_{n} c f$.

Proof. For some $c^{\prime} \in V(c)$ let $g=c c^{\prime}, h \in S(e, g)$ and $k \in S(f, g)$. By Lemma 1.1(iii) we may write $(e c)^{\prime}=c^{\prime} h e \in V(e c)$ and $(f c)^{\prime}=c^{\prime} k f \in V(f c)$. So $e c(e c)^{\prime}=e h$, $f c(f c)^{\prime}=f k,(e c)^{\prime} e c=c^{\prime} h c$ and $(f c)^{\prime}(f c)=c^{\prime} k c$. We will prove that $e h \mathscr{R}_{\pi} f k$ and $c^{\prime} h c \mathscr{L}_{n} c^{\prime} k c$. The proof that $c e \mathscr{H}_{\pi} c f$ is similar.

By condition (1), $(e h) \pi=r \pi$ where $r \in S(f h, f h)$. But $g h=h$ and $f g=f k g$ so $f k g h(f h)^{\prime} r=r=f k r$ for some $(f h)^{\prime} \in V(f h)$. Hence

$$
(f k) \pi *(e h) \pi=(f k) \pi * r \pi=(e h) \pi .
$$

Likewise $(e h) \pi *(f k) \pi=(f k) \pi$ so $e h \mathscr{R}_{\pi} f k$.

Again by condition (1) (he) $\pi=h \pi * e \pi=h \pi * f \pi=s \pi$ where $s \in S(h f, h f)$. So (hg) $\pi=(h e g) \pi=(h e) \pi * g \pi=s \pi * g \pi=t \pi$ where $t \in S(s g, s g)$. For $(h f)^{\prime} \in V(h f)$ and $(s g)^{\prime} \in V(s g)$ we have $s(h f)^{\prime} h f=s=s f$ so

$$
t=t(s g)^{\prime} s g=t(s g ') s f=t(s g)^{\prime} s f k g=t k g .
$$


Thus $(h g) \pi=t \pi *(k g) \pi=(h g) \pi *(k g) \pi$ and similarly $(k g) \pi *(h g) \pi=(k g) \pi$. We therefore have $h g \mathscr{L}_{\pi} \mathrm{kg}$. Now choose $p \in S(h g, k g)$. By Lemma 1.3(i), $p \in(k g) \pi$. Recalling that $g h=h$ and $g=c c^{\prime}$ it can be readily checked that $c^{\prime} p c \in S\left(c^{\prime} h c, c^{\prime} k c\right)$. Using condition (2)

$$
\begin{aligned}
\left(c^{\prime} k c\right) \pi & =\left(c^{\prime} k g c\right) \pi=\left(c^{\prime} p c\right) \pi=\left(c^{\prime} p h g c\right) \pi=\left(c^{\prime} p(g h) g c\right) \pi \\
& =\left(c^{\prime} p c\right) \pi *\left(c^{\prime} h c\right) \pi=\left(c^{\prime} k c\right) \pi *\left(c^{\prime} h c\right) \pi .
\end{aligned}
$$

Likewise $\left(c^{\prime} h c\right) \pi *\left(c^{\prime} k c\right) \pi=\left(c^{\prime} h c\right) \pi$. Hence $\left(c^{\prime} h c\right) \mathscr{L}_{\pi}\left(c^{\prime} k c\right)$.

\section{Normal partitions}

In this section normal partitions of $E_{S}$ will be characterized and the greatest congruences associated with these partitions will be determined.

THEOREM 2.1. Let $S$ be a regular semigroup and $P_{\pi}$ be an $N$-partition of $E_{S}$. Then $P_{\pi}$ is a normal partition and $\rho_{\pi}=\left\{(a, b) \in \mathscr{H}_{\pi}\right.$; for each $c \in S$, ca $\mathscr{H}_{\pi} c b$ and $\left.a c \mathscr{H}_{\pi} b c\right\}$ is the greatest congruence on $S$ that induces $P_{n}$.

PROOF. Clearly $\rho_{\pi}$ is symmetric, reflexive and compatible and by Lemma 1.3(ii) it is transitive. So $\rho_{\pi}$ is a congruence on $S$. If $(e, f) \in \pi$ then clearly $e \mathscr{H}_{\pi} f$ and by Lemma $1.4(e, f) \in \rho_{\pi}$. Conversely, if $(e, f) \in \rho_{\pi} \cap E_{S} \times E_{S}$ then $e \mathscr{H}_{\pi} f$. But then by Lemma 1.3(i), $S(e, f) \subseteq e \pi \cap f \pi$ so $e \pi=f \pi$. Thus $P_{\pi}$ is the normal partition of $E_{S}$ induced by $\rho_{\pi}$. Let $\tau$ be a congruence on $S$ that induces $P_{\pi}$. If $(a, b) \in \tau$ then $a \tau \mathscr{H} b \tau$ in $S / \tau$ and as noted before Lemma 1.4 then $a \mathscr{H}_{n} b$. Therefore $\rho_{\pi} \supseteq \tau$.

Since a normal partition of $E_{S}$ is an $N$-partition then:

CoROllary 2.2. $P_{\pi}$ is an $N$-partition of $E_{S}$ if and only if it is a normal partition of $E_{\boldsymbol{S}}$.

We can refine the description of $\rho_{\pi}$ by using the characterization of Hall (1973) of the greatest idempotent separating congruence $\mu$ on $S$. Using Lemma 1.2(ii), Hall's definition translates to the following:

$$
\begin{array}{r}
\mu=\left\{(a, b) \in \mathscr{H} ; \text { for some [any] } a^{\prime} \in V(a), b^{\prime} \in V(b)\right. \text { and each } \\
\text { idempotent } \left.e \leqslant a a^{\prime} \text { then } a^{\prime} e a=a^{\prime} a b^{\prime} a a^{\prime} e b\right\} .
\end{array}
$$

Note that if $\tau$ is a congruence on $S$, and $e, f \in E_{S}$ then $e \tau \leqslant f \tau$ in $S / \tau$ if and only if $(f e) \tau \mathscr{R} e \tau \mathscr{L}(e f) \tau$ in $S / \tau$. Hence for a normal partition $P_{n}$, define $e \pi \leqslant f \pi$ if and only if $(f e) \mathscr{R}_{n} e \mathscr{L}_{n}(e f)$. 
THEOREM 2.3. Let $S$ be a regular semigroup and $P_{\pi}$ be a normal partition of $E_{S}$. Then

$$
\begin{aligned}
\rho_{\pi}=\left\{(a, b) \in \mathscr{H}_{\pi} ; \text { for some }[a n y] a^{\prime} \in V(a), b^{\prime} \in V(b) \text { and each idempotent } e\right. \\
\\
\text { so that } e \pi \leqslant\left(a a^{\prime}\right) \pi \text { then } i \pi=j \pi \text { where } i \in S\left(a^{\prime} e a, a^{\prime} e a\right) \text { and } \\
\left.j \in S\left(a^{\prime} a b^{\prime} a a^{\prime} e b, a^{\prime} a b^{\prime} a a^{\prime} e b\right)\right\} .
\end{aligned}
$$

ProOF. Let $\sigma_{\pi}$ be the least congruence on $S$ inducing $P_{\pi}$ and let $\mu_{\pi}$ be the greatest idempotent separating congruence on $S / \sigma_{\pi}$. Then $(a, b) \in \rho_{\pi}$ if and only if $\left(a \sigma_{\pi}, b \sigma_{\pi}\right) \in \mu_{n}$. By the note preceding Lemma 1.4 we have $a \mathscr{H}_{n} b$ if and only if $a \sigma_{\pi} \mathscr{H} b \sigma_{n}$. For $e \in E_{S}, a \in S$ and $a^{\prime} \in V(a)$ we have $e \pi \leqslant\left(a a^{\prime}\right) \pi$ if and only if $e \sigma_{\pi} \leqslant\left(a a^{\prime}\right) \sigma_{\pi}$. Now suppose $e \in E_{S}, a, b \in S, a \mathscr{H}_{\pi} b, a^{\prime} \in V(a), b^{\prime} \in V(b)$ and $e \pi \leqslant\left(a a^{\prime}\right) \pi$. Then $\left(a^{\prime} e a\right) \sigma_{\pi} \in E_{S / \sigma_{\pi}}$. Also, since $a \sigma_{\pi} \mathscr{H} b \sigma_{\pi}$ it can be easily checked that ( $\left.a^{\prime} a b^{\prime} a a^{\prime} e b\right) \sigma_{\pi} \in E_{S / \sigma_{\pi}}$. Hence by Lemma 1.2(i), ( $\left.a^{\prime} e a\right) \sigma_{\pi}=\left(a^{\prime} a b^{\prime} a a^{\prime} e b\right) \sigma_{\pi}$ if and only if $S\left(a^{\prime} e a, a^{\prime} e a\right)$ and $S\left(a^{\prime} a b^{\prime} a a^{\prime} e b, a^{\prime} a b^{\prime} a a^{\prime} e b\right)$ are in the same $\pi$-class. The result follows from the definition of $\mu_{\pi}$.

\section{References}

A. H. Clifford (1974), The Fundamental Representation of a Regular Semigroup, Dept. of Maths., Tulane University, $65 \mathrm{pp}$.

R. Feigenbaum (1975), Kernels of regular semigroup homomorphisms, Doctoral dissertation, University of South Carolina.

T. E. Hall (1973), "On regular semigroups", J. Algebra 24, 1-24.

K. S. S. Nambooripad (1974), "Structure of regular semigroups I", Semigroup Forum 9, 354-363.

Department of Mathematics

University of Tasmania

Hobart, Tasmania

Australia 